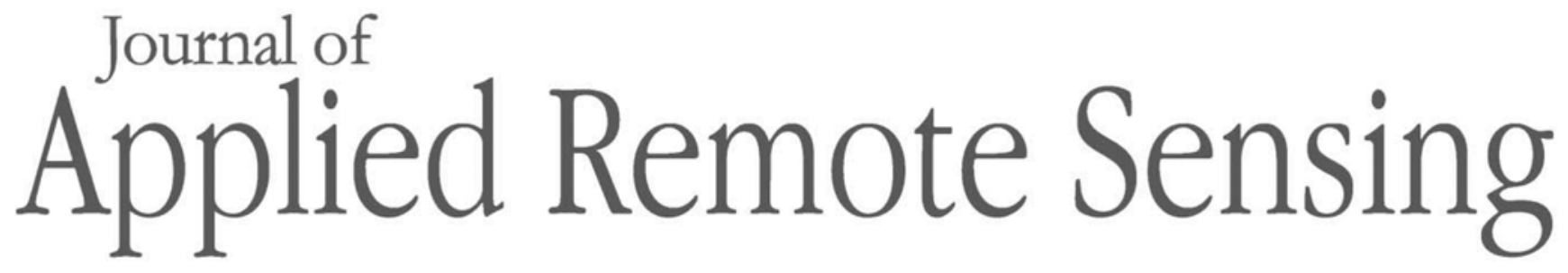

RemoteSensing.SPIEDigitalLibrary.org

\title{
Assessment of the application of cascade lasers to stand-off detection of alcohol vapors in moving cars
}

Jaroslaw Mlynczak

Jan Kubicki

Krzysztof Kopczynski

Jadwiga Mierczyk 


\title{
Assessment of the application of cascade lasers to stand-off detection of alcohol vapors in moving cars
}

\author{
Jaroslaw Mlynczak,* Jan Kubicki, Krzysztof Kopczynski, and \\ Jadwiga Mierczyk \\ Military University of Technology, Institute of Optoelectronics, Gen. S. Kaliskiego 2, \\ Warsaw 00-908, Poland
}

\begin{abstract}
The idea of using commercially available cascade lasers for stand-off detection of alcohol vapors in moving cars is presented. Special attention is paid to the optical characteristics of the car windowpanes for the monitoring as well as for the reference laser beams. A special experimental setup was built to investigate the idea. It is shown that using interband cascade lasers operating at 3.45- and 3.59- $\mu \mathrm{m}$ wavelengths, the alcohol vapors inside a car can be successfully detected, even in cars with different windowpanes. (C) The Authors. Published by SPIE under a Creative Commons Attribution 3.0 Unported License. Distribution or reproduction of this work in whole or in part requires full attribution of the original publication, including its DOI. [DOI: 10.1117/1.JRS.10.046010]
\end{abstract}

Keywords: alcolaser; stand-off detection of alcohol; application of cascade laser.

Paper 15897 received Dec. 24, 2015; accepted for publication Oct. 24, 2016; published online Nov. 14, 2016.

\section{Introduction}

Stand-off detection of alcohol in moving cars is a particular case of stand-off detection of vapors and gases described in papers. ${ }^{1-3} \mathrm{~A}$ device based on this technology can be potentially used to monitor the interior of a vehicle and finally contribute to eradication of the drunken drivers. As a result of such useful application, this technology enjoys popularity with many scientists and scientific institutions, which is shown in many patents. ${ }^{4-7}$ Much of the effort of the investigators were put into the measurement of the precise value of the alcohol concentration. ${ }^{6,7}$ Such approach does not seem to be appropriate for the reason that there are many phenomena disturbing precise measurement that cannot be foreseen and eliminated. These are, among others, car cooling system, open windows, window washing liquid (antifreeze), perfumes, or drunken passengers. Moreover, using carbon dioxide $\left(\mathrm{CO}_{2}\right)$, as in Ref. 7, also appears to be pointless for the very good reason that some passengers may be present in the car, who also exhales $\mathrm{CO}_{2}$. On account of that, the stand-off alcohol detection systems are not able to measure alcohol concentration in driver's blood, however, with absolute certainty, they are able to indicate the driver suspected of drinking alcohol. Such systems may be extremely useful and highly effective at minimizing the number of cars that should be checked by a policeman using more accurate hand-held equipment.

In most cases, the devices for stand-off detection of different gases utilize two radiation beams. ${ }^{8,9}$ The monitoring beam is absorbed by a monitored substance, whereas the reference beam should be close but outside of the absorption band. In case of the alcohol, the wavelength that is strongly absorbed is $3.39 \mu \mathrm{m}$. Not long ago, the only laser that could operate at this wavelength was $\mathrm{He}-\mathrm{Ne}$ laser and there was no other reference laser available on the market that could operate close to this wavelength. Using a commercially available semiconductor laser operating at $1.5 \mu \mathrm{m}$ as a reference was not suitable because of high difference in the transmissions of car windowpanes for 3.39 and $1.5 \mu \mathrm{m}$ caused by high difference in absorption coefficients. This, in turn, causes the ratio of these transmissions to depend strongly on cars, which are characterized by different windowpanes with different thickness and inclination.

*Address all correspondence to: Jaroslaw Mlynczak, E-mail: jaroslaw.mlynczak@wat.edu.pl 
If this ratio changes very much for different cars, the system does not work properly because any change is interpreted as a change in the alcohol concentration in the car cabin. The ideal situation would be if the absorption coefficients were the same and then the ratio of the transmissions was equal to 1 . To overcome this obstacle, the solution based on two 1.3- and 1.5- $\mu \mathrm{m}$ laser beams was proposed. ${ }^{8,10}$ This approach enabled the measuring of the thickness and inclination of the car windowpanes, which eliminated their impact on the results of the measurements. However, from a practical point of view, it was very difficult to develop such a system because the measured signals had to be determined with extremely high accuracy.

Currently, a completely new type of cascade laser operating around 3- to 4- $\mu \mathrm{m}$ wavelengths was developed and launched on the market. With the help of these lasers, the abovementioned problems can be overcome. In this paper, we present the idea of using cascade lasers for stand-off detection of alcohol vapors in moving cars as well as the results of the investigations of the experimental setup built to verify the idea.

\section{Basic Approach to Stand-Off Detection of Alcohol in Car Cabins}

The idea of stand-off detection of alcohol in a cabin with windowpanes is well known. ${ }^{9-12}$ The basic approach to this problem is shown in Fig. 1.

The collimated monitoring laser beam with intensity $I_{0}$ generated by the radiation source $L$ goes through windowpane $W_{1}$, where it is partially reflected and partially absorbed at both surfaces. Next, the beam $I_{1}$ goes through the cabin and the second windowpane $W_{2}$ and is finally absorbed by the detector $D$.

Assuming that the angles of incidence of the beam on the windowpanes are very small and neglecting multiple reflections inside the windowpanes, the beam intensity after going through the first windowpane can be described as

$$
I_{1}=I_{0}(1-r)^{2} e^{-\kappa_{\mathrm{W}} d_{\mathrm{W}}},
$$

where $r$ is Fresnel reflection coefficient of the windowpanes, $\kappa_{\mathrm{W}}-$ is the absorption coefficient of the beam inside the windowpanes, and $d_{\mathrm{W}}$ - is the thickness of the windowpanes.

If both windowpanes have the same $r, \kappa_{\mathrm{W}}$, and $d_{\mathrm{W}}$, and additionally, the absorption of the beam by alcohol inside the cabin and by possible dirt or water on the windowpanes is taken into account the beam intensity incident on the detector can be expressed as

$$
I=I_{1} e^{-\kappa_{\mathrm{a}} l_{\mathrm{c}}}(1-r)^{2} e^{-\kappa_{\mathrm{W}} d_{\mathrm{W}}}=I_{0}(1-r)^{4} e^{-2 \kappa_{\mathrm{W}} d_{\mathrm{W}}} e^{-\kappa_{\mathrm{a}} l_{\mathrm{c}}} e^{-\kappa_{\mathrm{d}} l_{\mathrm{d}}},
$$

where $\kappa_{\mathrm{a}}$ is the absorption coefficient of the alcohol in the cabin, $l_{\mathrm{c}}$ is the length of the cabin (distance between windowpanes), $\kappa_{\mathrm{d}}$ is the absorption coefficient of dirt or water, and $l_{\mathrm{d}}$ is the thickness of the dirt or water layer.

Dividing both sides of Eq. (2) by $I_{0}$ leads to

$$
\frac{I}{I_{0}}=(1-r)^{4} e^{-2 \kappa_{\mathrm{W}} d_{\mathrm{w}}} e^{-\kappa_{\mathrm{a}} l_{\mathrm{c}}} e^{-\kappa_{\mathrm{d}} l_{\mathrm{d}}}
$$

Introducing the total transmission of the cabin $T$, the transmission of the alcohol $T_{\mathrm{a}}$, the transmission of the cabin without the alcohol $T_{\mathrm{c}}$, and the transmission of dirt or water on the windowpanes $T_{d}$ is defined as

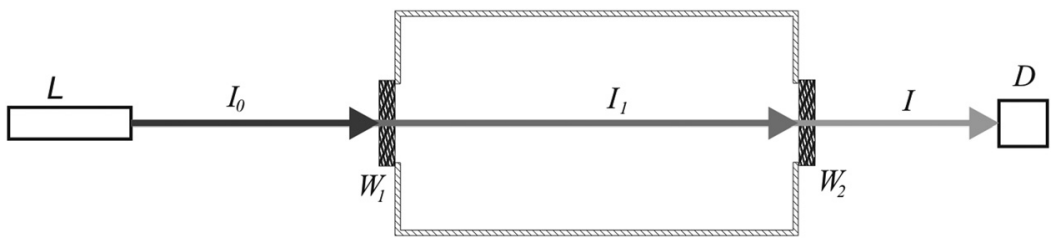

Fig. 1 Basic idea of stand-off detection of vapors and gases in a cabin with windowpanes. 


$$
\begin{gathered}
T=\frac{I}{I_{0}}, \\
T_{\mathrm{a}}=e^{-\kappa_{\mathrm{a}} \cdot l_{\mathrm{c}}}, \\
T_{\mathrm{c}}=(1-r)^{4} e^{-2 \kappa_{\mathrm{W}} d_{\mathrm{w}}}, \\
T_{\mathrm{d}}=e^{-\kappa_{\mathrm{d}} \cdot l_{\mathrm{d}}} .
\end{gathered}
$$

Equation (3) takes the form of

$$
T=T_{\mathrm{c}} \cdot T_{\mathrm{d}} \cdot T_{\mathrm{a}}
$$

Assuming linear characteristics of the detector $I / I_{0}=S / S_{0}$, Eq. (8) can be written as

$$
T=T_{\mathrm{c}} \cdot T_{\mathrm{d}} \cdot T_{\mathrm{a}}=\frac{S}{S_{0}},
$$

where $S$ is signal from the detector with the cabin and $S_{0}$ is signal from the detector without the cabin.

As it is shown in Sec. 3, the transmission of the laser beam by windowpanes is very small. It means that if there is no cabin (no car) between the radiation source and the detector, the signal incident on the detector is very high. On the other hand, if there is a cabin (car) with windowpanes, the signal is very low. This leads to the situation, where the dynamic of the intensity of the beam incident on the detector is very high. It is well known that to guarantee the linear characteristics of the detector (avoiding saturation), the dynamic of the signal incident on it should be small. It can be achieved by inserting an additional reference tube interchangeably with the cabin. In this case, the reference tube is placed between the radiation source and the detector and is removed when the cabin (car) crosses the radiation beam. The removal of the reference tube may be executed by a very fast-moving servomechanism. Certainly, there is possibility to eliminate the need for reference windowpanes by the use of a detector with higher linearity, however, according to the knowledge of the authors, there is no such detector available on the market at a reasonable price. Another issue is the car with open windowpanes. In this situation, the detector will saturate and the system will inform the user about this problem, which in turn means that the car needs to be checked by a police officer.

The transmission of the beam by this reference tube can be expressed as

$$
T_{\mathrm{r}}=\frac{S_{\mathrm{r}}}{S_{0}}
$$

where $S_{\mathrm{r}}$ is the signal from the detector with the reference tube.

Combining Eqs. (9) and (10) leads to

$$
\frac{T}{T_{\mathrm{r}}}=\frac{T_{\mathrm{c}}}{T_{\mathrm{r}}} \cdot T_{\mathrm{d}} \cdot T_{\mathrm{a}}=\frac{S}{S_{\mathrm{r}}}
$$

To minimize the impact of all obstacles (windowpanes, dirt, water) on the detection of alcohol in car cabin, a reference laser beam should be applied. Thus, the relation [Eq. (11)] can be expressed by two equations:

$$
\begin{aligned}
& \frac{T_{1}}{T_{\mathrm{r} 1}}=\frac{T_{\mathrm{c} 1}}{T_{\mathrm{r} 1}} \cdot T_{\mathrm{d} 1} \cdot T_{\mathrm{a} 1}=\frac{S_{1}}{S_{\mathrm{r} 1}}, \\
& \frac{T_{2}}{T_{\mathrm{r} 2}}=\frac{T_{\mathrm{c} 2}}{T_{\mathrm{r} 2}} \cdot T_{\mathrm{d} 2} \cdot T_{\mathrm{a} 2}=\frac{S_{2}}{S_{\mathrm{r} 2}},
\end{aligned}
$$

where 1 and 2 represent the monitoring beam and the reference beam, respectively. 
Combining Eqs. (12) and (13), one can achieve

$$
\frac{T_{\mathrm{c} 1}}{T_{\mathrm{c} 2}} \cdot \frac{T_{\mathrm{r} 2}}{T_{\mathrm{r} 1}} \cdot \frac{T_{\mathrm{d} 1}}{T_{\mathrm{d} 2}} \cdot \frac{T_{\mathrm{a} 1}}{T_{\mathrm{a} 2}}=\frac{S_{1}}{S_{2}} \cdot \frac{S_{\mathrm{r} 2}}{S_{\mathrm{r} 1}} .
$$

Introducing the following expressions, defining the relative transmission $\tau$ and the relative signal $s:$

$$
\tau_{\mathrm{c}}=\frac{T_{\mathrm{c} 1}}{T_{\mathrm{c} 2}}, \tau_{\mathrm{r}}=\frac{T_{\mathrm{r} 1}}{T_{\mathrm{r} 2}}, \tau_{\mathrm{d}}=\frac{T_{\mathrm{d} 1}}{T_{\mathrm{d} 2}}, \tau_{\mathrm{a}}=\frac{T_{\mathrm{a} 1}}{T_{\mathrm{a} 2}}, s=\frac{S_{1}}{S_{2}} \cdot \frac{S_{\mathrm{r} 2}}{S_{\mathrm{r} 1}}
$$

Equation (14) takes the form of

$$
\frac{\tau_{\mathrm{c}}}{\tau_{r}} \cdot \tau_{\mathrm{d}} \cdot \tau_{\mathrm{a}}=s,
$$

thus

$$
\tau_{\mathrm{a}}=\frac{1}{\tau_{\mathrm{d}}} \cdot \frac{\tau_{\mathrm{r}}}{\tau_{\mathrm{c}}} \cdot s .
$$

Introducing the following expression, defining the signal coefficient $\alpha$ :

$$
\alpha=\frac{1}{\tau_{\mathrm{d}}} \cdot \frac{\tau_{\mathrm{r}}}{\tau_{\mathrm{c}}} .
$$

Equation (17) can be expressed as

$$
\tau_{\mathrm{a}}=\alpha \cdot s .
$$

One could assume that the absorption coefficients of dirt or water for both beams are the same; however, it is not necessary the truth. In some situations, the dirt may consist of different chemical compounds characterized by selective transmission for different wavelengths. Thus, for a given cabin's dirt and water, $\alpha$ coefficient can be very easily determined by measuring the signals $S_{1}, S_{2}, S_{\mathrm{r} 1}$, and $S_{\mathrm{r} 2}$ for the reference tube and for the cabin without the alcohol $\left(\tau_{a}=1\right)$ and using the following equation:

$$
\alpha=\frac{1}{s}
$$

The problem appears, however, when unknown cabins with an unknown dirt and water layer are to be monitored. Then, the mean value of $\alpha$ should be determined on the basis of statistical investigations of different car cabins with different dirt and water layers.

\section{Spectral Characteristics}

The problem concerning transmission of car windowpanes described in the introduction as well as transmission of some chemical compounds that may be present in car cabins (alcohol, $\mathrm{H}_{2} \mathrm{O}$, and $\mathrm{CO}_{2}$ ) along with the spectra of lasers operating at 3.39-, 1.5-, 3.45-, and 3.59- $\mu \mathrm{m}$ wavelengths are illustrated in Fig. 2. Alcohol, $\mathrm{H}_{2} \mathrm{O}$, and $\mathrm{CO}_{2}$ transmission was measured using $10-\mathrm{cm}$ length tubes closed with glass plates filled with saturated alcohol vapors at $20^{\circ} \mathrm{C}\left(120 \mathrm{mg} / \mathrm{dm}^{3}\right)$, saturated water vapors at $20^{\circ} \mathrm{C}\left(17.3 \mathrm{mg} / \mathrm{dm}^{3}\right)$ and $\mathrm{CO}_{2}$ with $100 \%$ concentration, respectively. The results were corrected taking into account the transmission of the glass plates used in the experiments. The measurements were done with $4 \mathrm{~cm}^{-1}$ resolution. The figure shows only visual relation between investigated materials and does not express the real concentration of alcohol, $\mathrm{H}_{2} \mathrm{O}$, and $\mathrm{CO}_{2}$ that may appear in car cabins. In reality, one can expect much lower concentration. The only real characteristic is that of the car windowpane, where a typical Pilkington windowpane was used. One can see that for the He-Ne laser $(3.39 \mu \mathrm{m})$ as the monitoring beam and for the laser diode $(1.5 \mu \mathrm{m})$ as the reference beam, the transmissions of the car 


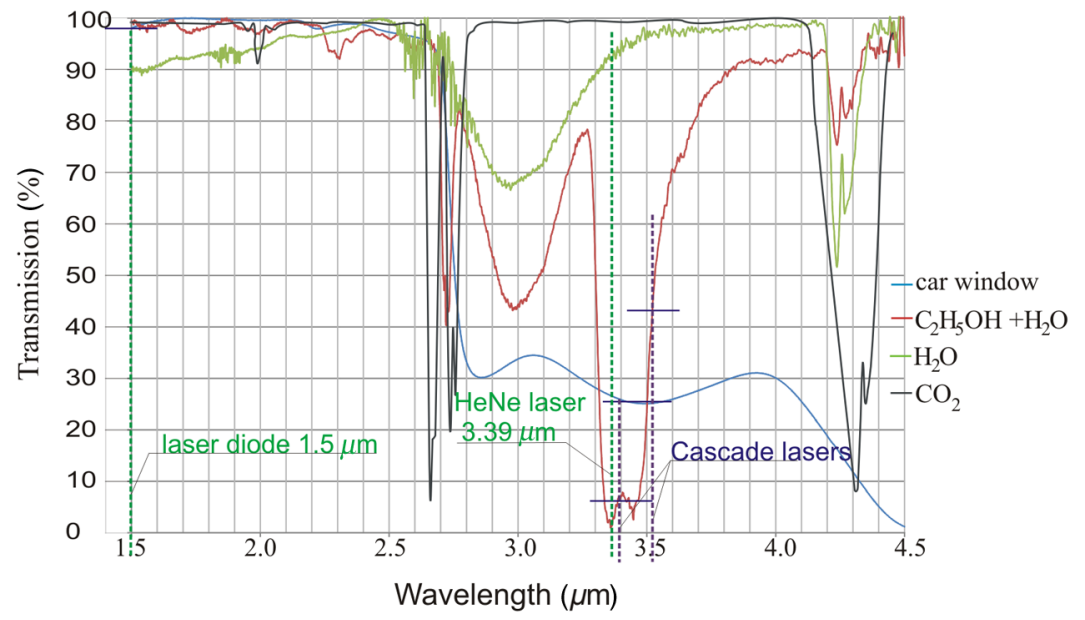

Fig. 2 Spectral characteristics of car windowpane (Pilkington), $\mathrm{CO}_{2}, \mathrm{H}_{2} \mathrm{O}$, alcohol, and lasers operating at 3.39-, 1.5-, 3.45-, and 3.59- $\mu \mathrm{m}$ wavelengths.

windowpane are completely different. It means that the change of the thickness of the car windowpane or its inclination has the same impact on the signal received by the detector as the change of the alcohol concentration in the car cabin.

By contrast, for the wavelengths $3.45 \mu \mathrm{m}$ (monitoring beam) and $3.59 \mu \mathrm{m}$ (reference beam), the transmissions of the car windowpanes are almost the same while the transmissions of alcohol are totally different. Even though the monitoring wavelength and the reference wavelength are very close to each other, there still may be some minor difference in the transmissions of the car windowpane, which results in some difference in $\alpha$ coefficient for different cars. In case of a small concentration of alcohol in a car cabin, such a situation may have vital impact on the results of detection. The transmissions of $\mathrm{H}_{2} \mathrm{O}$ and $\mathrm{CO}_{2}$ in case of their much lower concentration (normal conditions) are almost the same; so, the impact of their amount on the result of the measurement of the alcohol concentration is also minimal.

In case of high concentration of water (high humidity, snow, ice), the situation is much more complicated. It is not only because of strong absorption by water but also because water actually has a very strong and similar absorption spectra for the two wavelengths used. It means that in conditions of high humidity, the detection of alcohol vapors characterized by low concentration is not possible. So, the device based on this technology cannot work in all environmental conditions. There are also many other substances, such as dirt that may have a small impact on the measurements. The cars with such dirt on the windowpanes, as well as cars with covered side windowpanes and opened side windowpanes, will be considered as suspected and stopped to undergo detailed control by a policeman.

The water background issue as well as dirt are much less important in normal conditions than the car windowpane parameters. So, it is reasonable not to take them into account separately but as an uncertainty of the measurements. However, much meticulous investigations of the impact of different substances on the detection of alcohol vapor in moving cars are necessary.

To determine the importance of the problem with car windowpanes, the transmission of eight different car windowpanes was examined. To investigate the impact of the water on the transmission, the windowpane 1 was also covered by steam. It was put into a refrigerator and cooled down to $-4^{\circ} \mathrm{C}$. When it was taken out, water vapor condensed on it. The results of measurements are shown in Fig. 3: 1, windowpane of 2-mm thickness; 2, tampered Pilkington car windowpane of 3.15-mm thickness; 3, not tempered Pilkington car windowpane of 3.15-mm thickness; 4, windowpane of 2.9-mm thickness; 5, dimmed tampered Pilkington car windowpane of 3.15$\mathrm{mm}$ thickness; 6, windowpane of 3.9-mm thickness; 7, dimmed tampered Soliver car windowpane of 4-mm thickness; 8 , windowpane of 4-mm thickness; 1a, steamed-up windowpane 1.

The measured transmission of the windowpanes for the cascade lasers at 3.45 and $3.59-\mu \mathrm{m}$ wavelength is presented in Table 1 . The transmission of $3.45-\mu \mathrm{m}$ wavelength by single windowpane was denoted as $T_{\mathrm{s} 1}$, the transmission of $3.59-\mu \mathrm{m}$ wavelength by single windowpane as $T_{\mathrm{s} 2}$, 


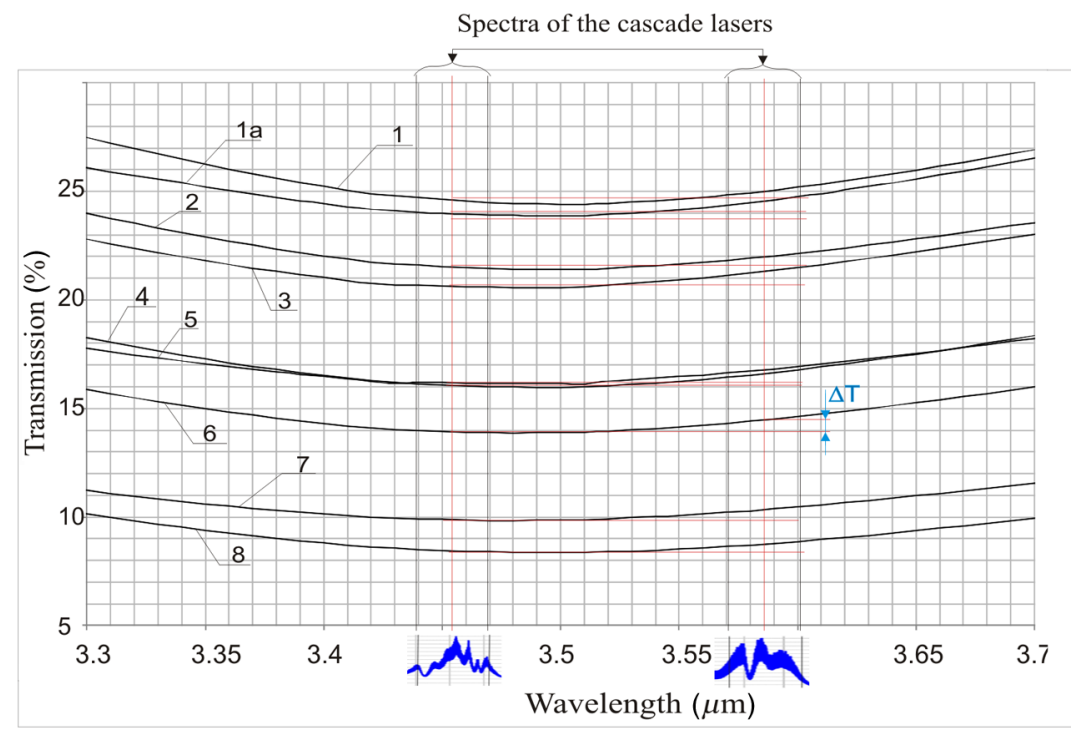

Fig. 3 Spectral characteristics of car windowpanes: 1, windowpane of 2-mm thickness; 2, tampered Pilkington car windowpane of 3.15-mm thickness; 3, not tempered Pilkington car windowpane of 3.15-mm thickness; 4, windowpane of 2.9-mm thickness; 5, dimmed tampered Pilkington car windowpane of 3.15-mm thickness; 6 , windowpane of 3.9-mm thickness; 7, dimmed tampered Soliver car windowpane of 4-mm thickness; 8, windowpane of 4-mm thickness; 1a, steamed up windowpane 1.

Table 1 Measured transmission of the windowpanes for 3.45- and 3.59- $\mu \mathrm{m}$ wavelength and calculated transmission of the steam.

\begin{tabular}{lcccccccccc}
\hline \hline Windowpane & 1 & 2 & 3 & 4 & 5 & 6 & 7 & 8 & $1 \mathrm{a}$ & Steam \\
\hline$T_{\mathrm{s} 1}$ & 0.2470 & 0.2159 & 0.2067 & 0.1614 & 0.1620 & 0.1400 & 0.0993 & 0.0852 & 0.2402 & 0.9723 \\
$T_{\mathrm{s} 2}$ & 0.2482 & 0.2181 & 0.2112 & 0.1643 & 0.1664 & 0.1432 & 0.1022 & 0.0865 & 0.2436 & 0.9816 \\
$T_{1}$ & 0.0610 & 0.0466 & 0.0427 & 0.0261 & 0.0262 & 0.0196 & 0.0099 & 0.0073 & 0.0577 & 0.9453 \\
$T_{2}$ & 0.0616 & 0.0476 & 0.0446 & 0.0270 & 0.0277 & 0.0205 & 0.0104 & 0.0075 & 0.0594 & 0.9636 \\
$T_{2}-T_{1}$ & 0.0006 & 0.001 & 0.0019 & 0.0009 & 0.0015 & 0.0009 & 0.0005 & 0.0002 & 0.0017 & 0.0183 \\
\hline \hline
\end{tabular}

the transmission of $3.45-\mu \mathrm{m}$ wavelength by double windowpane as $T_{1}$, and the transmission of $3.59-\mu \mathrm{m}$ wavelength by double windowpane as $T_{2}$. Taking into account the transmission of the windowpane 1 with and without steam, the transmission of the steam was also calculated and presented in Table 1. The calculations were done according to the following equations:

$$
T_{1}=T_{\mathrm{s} 1}^{2}, T_{2}=T_{\mathrm{s} 2}^{2}, T_{\mathrm{s} 1}(\text { steam })=\frac{T_{\mathrm{s} 1}(1 a)}{T_{\mathrm{s} 1}(1)}, T_{\mathrm{s} 2}(\text { steam })=\frac{T_{\mathrm{s} 2}(1 a)}{T_{\mathrm{s} 2}(1)}
$$

One can see that the difference of the transmissions for both beams $T_{2}-T_{1}$ is different for different windowpanes, however, it is very small. The smaller $T_{2}-T_{1}$, the smaller impact of different windowpanes on the results of measurements of the alcohol concentration.

\section{Determination of $\alpha$ Coefficient}

To determine the value of $\alpha$ coefficient for different car windowpanes and dirt, a special experimental setup was built, which is shown in Fig. 4. Two multimode interband cascade lasers, IF3420CM1 and IF3550CM1, developed by Thorlabs company operating at 3.45- and 


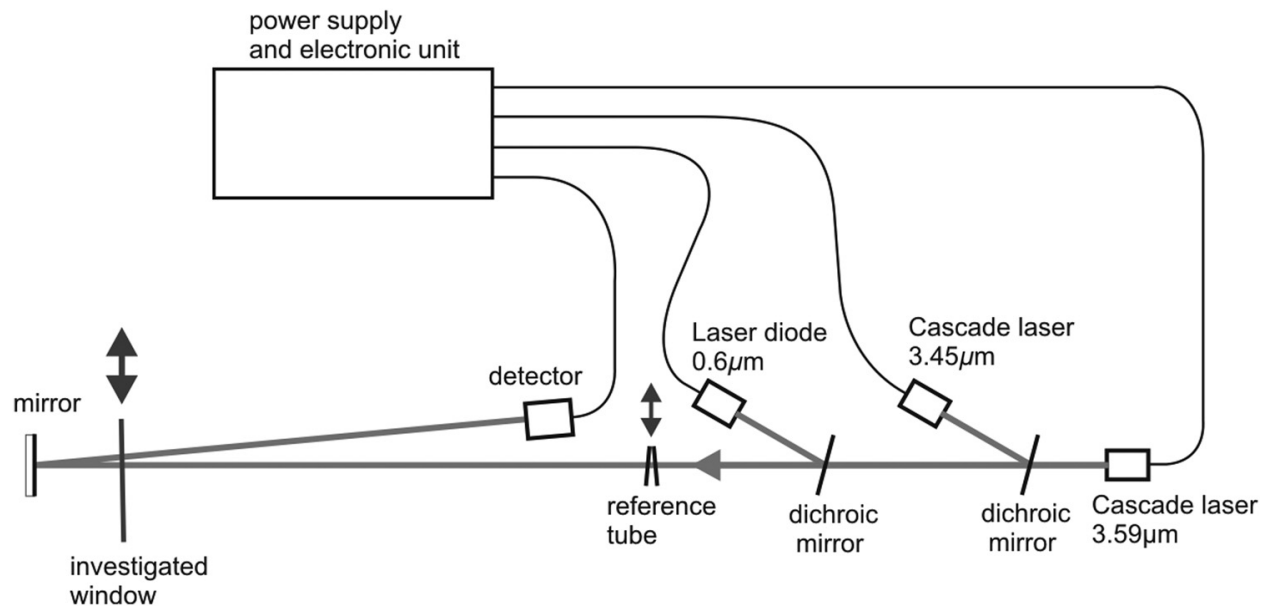

Fig. 4 Experimental setup used to determine $\alpha$ coefficient.

$3.59-\mu \mathrm{m}$ wavelengths with output power $35 \mathrm{~mW}$ were used. They were driven by an ITC4005QCL power supply, also developed by Thorlabs company. The lasers worked in pulsed mode with pulse repetition rate 5 and $8 \mathrm{kHz}$ with current amplitude equal to $0.5 \mathrm{~A}$. The beams went through the reference tube made of windowpane of 2.9-mm thickness (shown in Fig. 3 as windowpane 4) or the investigated windowpane and were detected by the TE-cooled PbSe detector P4639 developed by Hamamatsu company, characterized by $9-\mathrm{mm}^{2}$ aperture. The small angle equal to $87 \mathrm{mrad}$ between the windowpanes constituting the reference tube eliminated the interference that could disturb the signal. Two dichroic mirrors were used to integrate three laser beams into one. The first dichroic mirror was characterized by $50 \%$ transmission for both 3.45 and $3.59-\mu \mathrm{m}$ wavelengths, whereas the second one was characterized by $100 \%$ transmission for 3.45 and $3.59-\mu \mathrm{m}$ wavelengths and $100 \%$ reflection for $0.6-\mu \mathrm{m}$ wavelength. The mirror reflecting the signal onto the detector was characterized by $100 \%$ reflection for all wavelengths. The signals from the detector were analyzed by a special electronic unit, where the amplitude of the signal was measured. After 1000 measurements, the signal was averaged. The laser diode operating at $0.6-\mu \mathrm{m}$ in $\mathrm{cw}$ mode was used to align the system.

To simulate the cabin without alcohol, the beams went through the investigated windowpanes two times, as can be seen in Fig. 4. Using Eq. (18), $\alpha$ coefficient was calculated. All the previously investigated windowpanes were examined as well as windowpane 1 covered with steam, graphite dust, and soil dust denoted as $1_{\mathrm{st}}, 1_{\mathrm{gr}}$, and $1_{\mathrm{so}}$, respectively. The calculations of $\alpha$ coefficient for the layers of steam, graphite dust, and soil dust were done according to the following equations:

$$
\alpha(\mathrm{st})=\frac{\alpha\left(1_{\mathrm{st}}\right)}{\alpha(1)}, \alpha(\mathrm{gr})=\frac{\alpha\left(1_{\mathrm{gr}}\right)}{\alpha(1)}, \alpha(\mathrm{so})=\frac{\alpha\left(1_{\mathrm{so}}\right)}{\alpha(1)} .
$$

The results of the calculations are presented in Table 2. The layers of steam, graphite dust, and soil dust were designated as st, gr, and so. The average value of $\alpha$ coefficient was calculated to be $\bar{\alpha}=1.030$ with standard deviation $\delta=0.037$.

Table 2 Results of calculations of $\alpha$ coefficient.

\begin{tabular}{lcccccccccccccc}
\hline \hline Windowpane & 1 & 2 & 3 & 4 & 5 & 6 & 7 & 8 & $1_{\mathrm{st}}$ & $1_{\mathrm{gr}}$ & $1_{\mathrm{so}}$ & st & $\mathrm{gr}$ & so \\
\hline$\alpha$ & 1.052 & 1.047 & 1.028 & 1.000 & 0.979 & 1.003 & 0.985 & 1.003 & 1.070 & 1.088 & 1.078 & 1.017 & 1.034 & 1.025 \\
\hline \hline
\end{tabular}




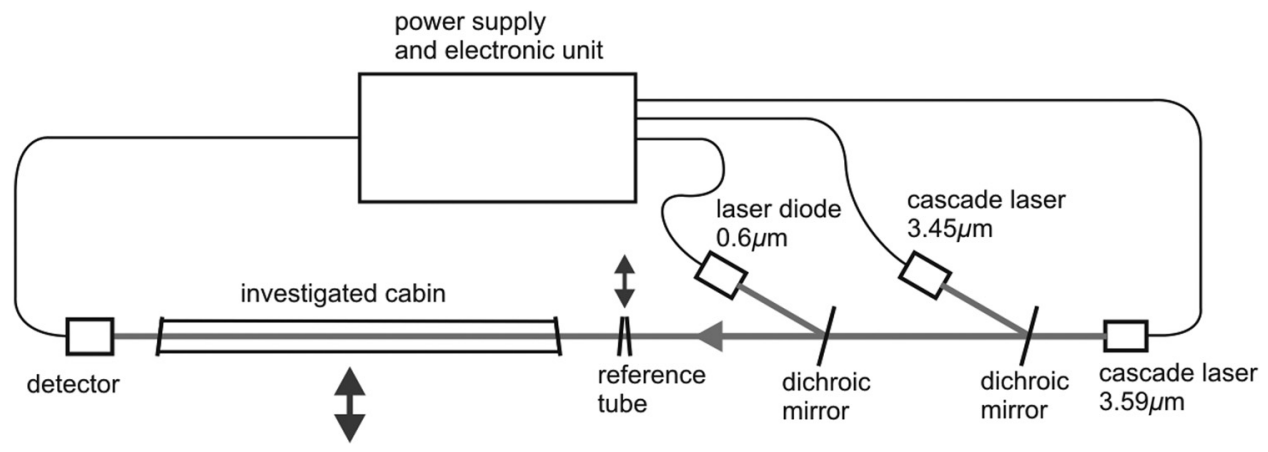

Fig. 5 Experimental setup for the investigation of relative transmission of alcohol in the cabin.

\section{Investigation of the Transmission of the Car Cabins with Different Alcohol Concentrations}

The setup for the investigation of the transmission of the cabin with different concentration of alcohol is shown in Fig. 5. It is almost the same as in Fig. 4 except for the investigated windowpane, which was replaced by the cabin with alcohol vapors and for the mirror, which was removed. The cabin was made of $140-\mathrm{cm}$ long plastic tube with the diameter equal to $10 \mathrm{~cm}$ closed with windowpanes (windowpane 4).

Before each measurements, a special container $(2.4 \mathrm{~cm}$ wide and $140 \mathrm{~cm}$ long) filled with a water solution of alcohol with different concentrations was inserted into the cabin, where the temperature was $20^{\circ} \mathrm{C}$. In such condition, the alcohol started to evaporate increasing the concentration of alcohol vapor in time. The state of equilibrium was reached after about $20 \mathrm{~min}$ every time the measurements were carried out. The concentration of alcohol vapors in the cabin $C_{\mathrm{av}}$ for each concentration of water solution of alcohol was determined on the basis of Henry's law. ${ }^{13}$ The calculated relative transmission of alcohol in the cabin $\tau_{\mathrm{a}}$ for different concentrations, according to Eqs. (19) and (20), is presented in Table 3 and Fig. 6.

Table 3 Calculated relative transmission of alcohol $\tau_{\mathrm{a}}$ for different concentration.

\begin{tabular}{lccccc}
\hline \hline Alcohol concentration $C_{\mathrm{av}}\left(\mathrm{mg} / \mathrm{dm}^{3}\right)$ & 0.0 & 0.1 & 0.2 & 0.3 & 0.4 \\
\hline$\tau_{\mathrm{a}}$ & 1.000 & 0.925 & 0.843 & 0.782 & 0.698 \\
\hline \hline
\end{tabular}

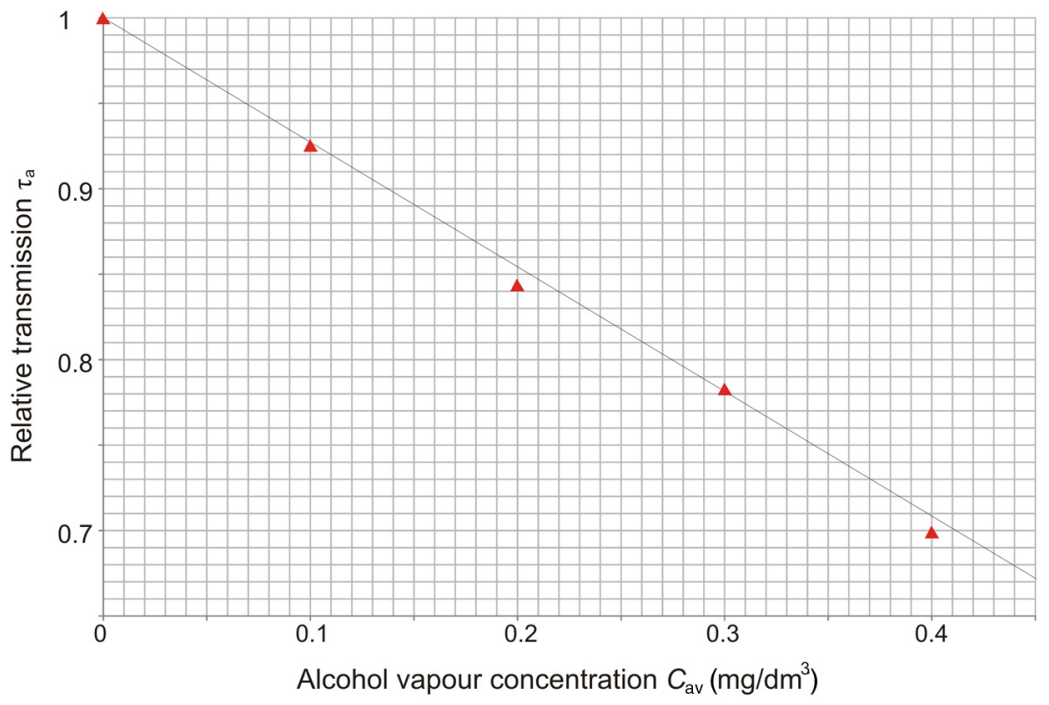

Fig. 6 Relative transmission $\tau_{\mathrm{a}}$ in function of alcohol concentration. 
The relation between the relative alcohol transmission $\tau_{\mathrm{a}}$ and the alcohol concentration $C_{\mathrm{av}}$ can be approximated by the following linear function:

$$
\tau_{\mathrm{a}}=1-0.725 \cdot C_{\mathrm{av}}\left[\frac{\mathrm{mg}}{\mathrm{dm}^{3}}\right] .
$$

The relation [Eq. (23)] is universal for the given length of the cabin and does not depend on the type of the windowpanes because $\alpha$ coefficient was measured for $C_{\mathrm{av}}=0$ for which $\tau_{\mathrm{a}}$ is equal to 1 .

\section{Investigation of Alcohol Detection in Car Cabins with Different Windowpanes}

The $\alpha$ coefficient is not known for the cars that will be checked in a real environment but its average value for a given number of cars can be determined and used to examine the subsequent cars after installation of the system by a road. Using the average value of $\alpha$ coefficient $\bar{\alpha}=1.030$ and its standard deviation $\delta=0.037$, relative alcohol transmission $\tau_{\mathrm{a}}$ for different $C_{\mathrm{av}}$ can be calculated using Eq. (19). The results of the calculations are presented in Table 4 and graphically shown in Fig. 7, where the measured points were approximated by a linear function. Because the same windowpane 4 was used to build the cabin as well as the reference tube; thus, the exact value of $\alpha$ coefficient is equal to 1 . The calculations for $\alpha=1$ are also shown in Table 4 and Fig. 7.

Table 4 Relative alcohol transmission $\tau_{\mathrm{a}}$ for $\alpha, \bar{\alpha}$ as well as for $\bar{\alpha}-\delta$ and $\bar{\alpha}+\delta$.

\begin{tabular}{lcccc}
\hline \hline$C_{\mathrm{av}}\left(\mathrm{mg} / \mathrm{dm}^{3}\right)$ & $\tau_{\mathrm{a}}=\alpha \cdot s$ & $\bar{\tau}_{\mathrm{a}}=\bar{\alpha} \cdot s$ & $\tau_{\mathrm{a}}=(\bar{\alpha}-\delta) \cdot s$ & $\tau_{\mathrm{a}}=(\bar{\alpha}+\delta) \cdot s$ \\
\hline 0.0 & 1.000 & 1.007 & 0.970 & 1.043 \\
0.1 & 0.924 & 0.931 & 0.897 & 0.964 \\
0.2 & 0.843 & 0.849 & 0.818 & 0.879 \\
0.3 & 0.782 & 0.787 & 0.759 & 0.816 \\
0.4 & 0.698 & 0.703 & 0.678 & 0.728 \\
\hline \hline
\end{tabular}

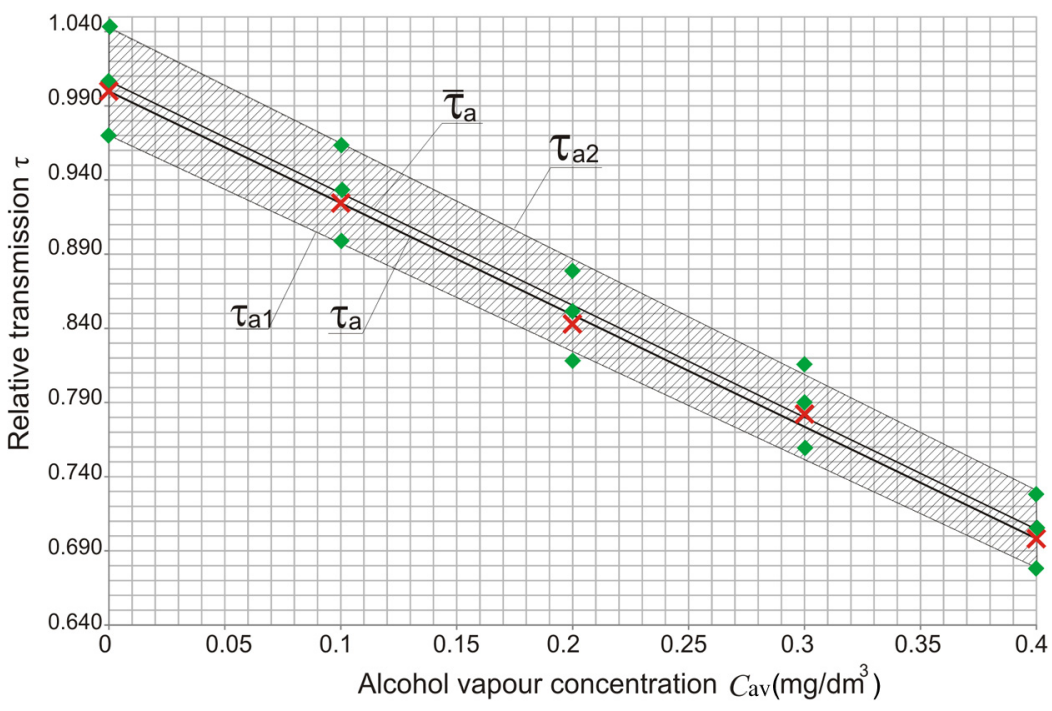

Fig. 7 Relative alcohol transmission $\tau_{\mathrm{a}}$ in function of $C_{\mathrm{av}}$. 
For any object (car or cabin), the relative transmission $\tau_{\mathrm{a}}$ for a given concentration of alcohol vapor $C_{\mathrm{av}}$ is described by Eq. (23), which after transformation takes the form of

$$
C_{\mathrm{av}}=\frac{1-\tau_{\mathrm{a}}}{0.725}
$$

Taking into account standard deviation, the real value of $\alpha$ coefficient is in the range of $\bar{\alpha}-\delta<$ $\alpha<\bar{\alpha}+\delta$ with the probability of $68 \%$. It means that in this case, $0.993<\alpha 1.067$. These boundaries relate to $\tau_{\mathrm{a} 1}=\alpha_{1} \cdot S$ and $\tau_{\mathrm{a} 2}=\alpha_{2} \cdot S$, as shown in Fig. 7, where $\alpha_{1}=0.993$, $\alpha_{2}=1.067, s=\frac{S_{1}}{S_{2}} \cdot \frac{S_{2 \mathrm{w}}}{S_{1 \mathrm{w}}}=\frac{\tau_{\mathrm{a}}}{\alpha}$

In the target device, the electronic unit will calculate the measured concentration of alcohol vapor $C_{\mathrm{av}}^{m}$ on the basis of the measured signals and average value of $\alpha$ coefficient according to the following formula:

$$
C_{\mathrm{av}}^{m}=\frac{1-\tau_{\mathrm{a}}^{m}}{0.725}=\frac{1-\bar{\alpha} \cdot s}{0,725} .
$$

However, the result will be approximate, mainly because of the assumed average value of $\alpha$. On the basis of preliminary experiments, the impact of the stability of lasers, detectors, and electronic units, is much smaller, however, it needs to be investigated more deeply in farther work. Thus, the real concentration of alcohol vapor $C_{\mathrm{av}}$ will be in the range of $C_{\mathrm{av} 1} \leq C_{\mathrm{av}} \leq$ $C_{\text {av2 }}$ with already mentioned probability of $68 \%$.

On the basis of Eq. (24), the boundaries of $C_{\mathrm{av}}$ for a given measured value of $C_{\mathrm{av}}^{m}$ are

$$
C_{\mathrm{av} 1}=\frac{1-\alpha_{1} \cdot s\left(C_{\mathrm{av}}^{m}\right)}{0.725}, C_{\mathrm{av} 2}=\frac{1-\alpha_{2} \cdot s\left(C_{\mathrm{av}}^{m}\right)}{0.725} .
$$

Using Eqs. (19) and (23), one can get $s\left(C_{\mathrm{av}}^{m}\right)=\frac{1-0.725 \cdot C_{\mathrm{av}}^{m}}{\bar{\alpha}}$, and assuming that $\bar{\alpha}=1.030$, Eq. (26) can be written as

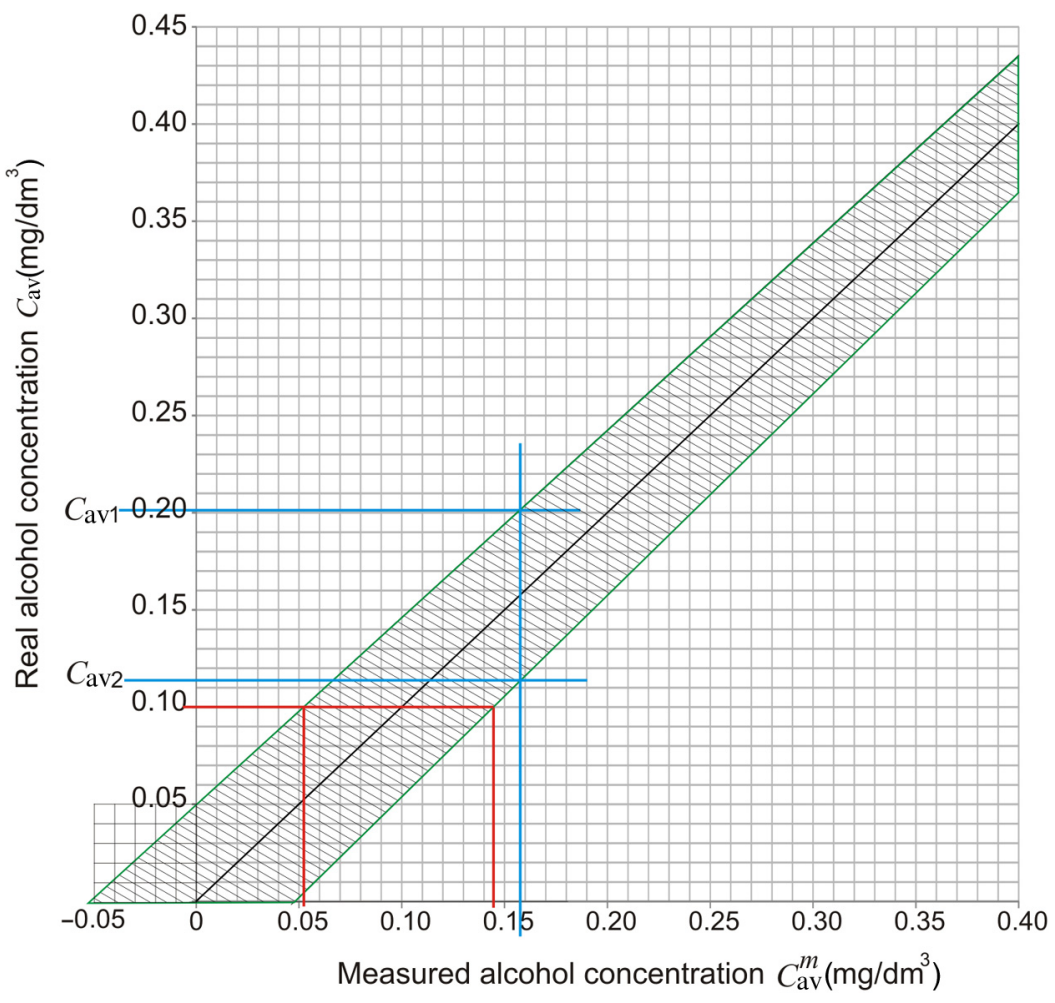

Fig. 8 Illustration of the possibility of stand-off measurement of the real value of alcohol vapor concentration in car cabins. 


$$
\begin{aligned}
& C_{\mathrm{av} 1}=1.379-0.964 \cdot\left(1.379-C_{\mathrm{av}}^{m}\right), \\
& C_{\mathrm{av} 2}=1.379-1.036 \cdot\left(1.379-C_{\mathrm{av}}^{m}\right) .
\end{aligned}
$$

The relations Eq. (27) and Eq. (28) are presented in Fig. 8.

On the basis of the analysis shown in Fig. 8, one can conclude that for the measured value of alcohol concentration $0.158 \mathrm{mg} / \mathrm{dm}^{3}$, its real value is in the range of $0.114 \div 0.202 \mathrm{mg} / \mathrm{dm}^{3}$ with the probability of $68 \%$. Moreover, there is no alcohol in the cabin with the probability of $68 \%$ when the measured value of alcohol concentration is $C_{\mathrm{av}}^{m}<-0.05 \mathrm{mg} / \mathrm{dm}^{3}$. Very important issue is the detection of alcohol in the exhaled air when the concentration is $0.1 \mathrm{mg}$ in $1 \mathrm{dm}^{3}$, which is the legal limit of alcohol vapor concentration in many countries. ${ }^{14,15}$ As can be seen in Fig. 8, the real alcohol concentration $0.1 \mathrm{mg} / \mathrm{dm}^{3}$ relates to the measured value in the range of 0.052 to $0.144 \mathrm{mg} / \mathrm{dm}^{3}$ with the probability of $68 \%$.

\section{Conclusions}

On the basis of the presented investigations and analysis, it can be concluded that the cascade lasers operating at 3.45- and 3.59- $\mu \mathrm{m}$ wavelengths can be successfully used in the systems for stand-off detection of alcohol concentration in moving cars. Even though the windowpanes used in the experiments were characterized by different parameters (thickness, manufacturing technology, tempering, dust, steam), the investigations show that $\alpha$ coefficient does not change much and the detection of alcohol vapor at the level of $0.1 \mathrm{mg} / \mathrm{dm}^{3}$ is still possible.

Nevertheless, more thorough investigations of the stability of lasers, detectors, and electronic unit as well as the water and dirt issues, need to be carried out and presented in another publication.

\section{References}

1. R. M. Silverstein, Spectrometric Identification of Organic Compounds, John Wiley \& Sons, New York (1991).

2. W. Volland, Organic Compound Identification Using Infrared Spectroscopy, Bellevue Community College, Washington (1999).

3. Z. Mierczyk et al., Optoelectronic Monitoring of Industrial Waste Management, EKOMILITARIS, Zakopane, Poland (2004).

4. N. Shuji, "Alcohol detector in vehicle," Patent No. JP2000230900 (A) (2000).

5. O. Ershov, A. Nadezdinskii, and A. Berezin, "Remote gas molecule detector," Patent No. US2003/0160173A1 (2003).

6. J. Kubicki et al., "Device for detection of alcohol vapours in moving vehicles," Patent No. P389627 (2009).

7. P. Kluczynski and S. Lundqvist, "Method and apparatus for remote detection of alcohol vapours in the atmosphere," Patent No. EP20130002759 (2013).

8. J. Kubicki, J. Mlynczak, and K. Kopczynski, "Application of modified difference absorption method to stand-off detection of alcohol in simulated car cabins," J. Appl. Remote Sens. 7(1), 073529 (2013).

9. E. V. Browell, S. Ismail, and W. B. Grant, "Differential absorption lidar (DIAL) measurements from air and space," Appl. Phys. B Lasers Opt. 67(4), 399-410 (1998).

10. Z. Mierczyk et al., "Device for stand-off detection of vapours and gases using DIAL method in cabins and chambers with windows," Patent No. P398513 (2012).

11. J. Mlynczak, J. Kubicki, and K. Kopczynski, "Stand-off detection of alcohol in car cabins," J. Appl. Remote Sens. 8(1), 083627 (2014).

12. J. Młyńczak, J. Kubicki, and K. Kopczyński, "Possibilities of stand-off detection of alcohol in car cabins," Przegl. Elektrotech. 2014(8), 53-56 (2014).

13. W. M. Meylan and P. H. Howard, "Bond contribution method for estimating Henry's law constants," Environ. Toxicol. Chem. 10, 1283-1293 (1991). 
14. J. Mlynczak et al., "Possibilities of Stand-Off Detection of Alcohol in Car Cabins with Windows," pp. 53-58, IAPGOS, Lublin, Poland (2015).

15. The lower chamber of the Polish parliament, "Polish act about up-bringing in sobriety and alcoholism prevention," http://isap.sejm.gov.pl/DetailsServlet?id=WDU20021471231 (26 October 1982).

Jaroslaw Mlynczak received his MSc in 2002 and PhD in 2008 from the Military University of Technology, Warsaw, Poland, where he currently works as a scientist. His research centers on investigation of new active media and new nonlinear absorbers for UV, VIS, IR, and "eye-safe" microchip lasers as well as development of microchip lasers for stand-off detection systems. He also participates in research concerning the detection of biological and chemical agents in the environment as well as biometric identification of people. He is an author and coauthor of many scientific and conference papers.

Jan Kubicki is a graduate from the Military University of Technology, Warsaw, Poland. In 1980, he received his $\mathrm{PhD}$ in the field of molecular lasers. Currently, he works at the Institute of Optoelectronics of Military University of Technology. He is an author and coauthor of many scientific papers in the fields of laser physics, laser spectroscopy, and high power laser systems.

Krzysztof Kopczynski received his MSc in solid-state physics and quantum electronics and $\mathrm{PhD}$ in the field of laser physics from the Military University of Technology. He is a director of the Institute of Optoelectronics of Military University of Technology. He is a specialist in the fields of solid-state lasers, microchip lasers, and laser devices for stand-off detection. He is an author and coauthor of multiple scientific and conference papers.

Jadwiga Mierczyk received her MSc from Silesian University in Poland. She is a specialist in the field of spectrometry and photometry. She is an author and coauthor of many scientific and conference papers. 